Objectives: To investigate and compare etanercept monotherapy versus combination with SDMARDs in RA patients regarding survival on drug, efficacy, quality of life and reason for discontinuation.

Methods: Observational, open, long-term study of patients from the ATTRA registry starting the ETN therapy since 2010 either on monotherapy or in combination with sDMARDs $(n=605) .461$ patients $(80.7 \%)$ were females, rheumatoid factor was positive in $429(76.6 \%)$, anti CCP antibodies in 412 $(74.5 \%)$ patients. The following groups of patients were assessed: Etanercept monotherapy (ETN-mono, $\mathrm{n}=83$ ), combination with methotrexate (Combo-MTX, $\mathrm{n}=378$ ), combination with other sDMARDs (Combo-other sDMARDs, $\mathrm{n}=110$ ). Mean \pm SD and absolute/relative frequencies were used to describe continuous and categorical variables, respectively. P-value of Pearson Chi-square test and Kruskal-Wallis test is given when assessing differences between groups in categorical and continuous variables. Results are presented as hazard ratio (HR) with corresponding $p$-value. Statistical evaluation was processed by IBM SPSS Statistics ver. 24

Results: In the first measurement of activity after 3 months, there were significantly lower disease activity in both Combo groups compared to ETN mono ( $3.2 \pm 0.1$ resp. $2.9 \pm 0.1$ versus $3.7 \pm 0.2, p<0.001$ ). However since the month 6 the differences lost their significance and the disease activity were comparable in all groups up to the month 24

The $H A Q$ value was significantly higher already at the time of ETN onset at month 0 in the ETN-mono group (1.7 versus 1.4 resp. $1.5, p<0.001$ ) and stayed significantly higher at the month 3 and 12 .

The hazard ratio (HR) for ETN discontinuation was higher in ETN mono group compared to Combo-MTX (HR=1.330) and also compared to Combo-other sDMARDs ( $H R=1.098)$, even if the differences did not reach statistical significance $(p=0.131$, resp. 0.671). Similar trend was evident, if the etanercept was given in the first and any subsequent line of biological therapy. The HR for ETN discontinuation was also numerically higher in Combo-other sDMARDs than in Combo MTX group (HR=1.211, $\mathrm{p}=0.244$ ).

The patients on ETN mono have higher risk of discontinuation due to inefficacy compared to Combo-MTX (HR=1.415) and also to Combo-other sDMARDs $(H R=1.670)$. The patients of combo-other sDMARDs group had lower risk of discontinuation due to inefficacy also in comparison with combo-MTX ( $H R=0.847)$. None of the differences reach statistical significance.

The patients on ETN mono have higher risk of discontinuation due to adverse event compared to Combo-MTX and also to Combo-other sDMARDs. This trend is higher, if ETN is given in the subsequent line of biological therapy, but not in first line. Nevertheless like in other situations, the differences showed some trend, but were not statistically significant.

Conclusions: Results support the evidence of advantages of ETN combination with methotrexate - quicker onset of efficacy and give some evidence of better survival on etanercept therapy in combination with methotrexate.

Acknowledgements: Supported by AZV 15-28659A

Disclosure of Interest: None declared

DOI: 10.1136/annrheumdis-2017-eular.2583

\section{AB0388 RESULTS AT 3 YEARS OF AN OPTIMIZATION GUIDELINE OF BIOLOGICAL THERAPIES IN RHEUMATOID ARTHRITIS. CREATE RECORD RESULTS}

P. Font ${ }^{1}$, M.C. Castro ${ }^{2}$, M. Romero ${ }^{2}$, R. Ortega ${ }^{2}$, J. Calvo ${ }^{2}$, D. Ruiz ${ }^{2}$, M. Cardenas ${ }^{2}$, R. Alejandre ${ }^{2}$, P. Carreto ${ }^{3}$, E. Collantes ${ }^{2} .{ }^{1}$ Hospital Reina Sofia Imibic-Universidad de Córdoba; ${ }^{2}$ Hospital Reina Sofia - Imibic-Universidad de Córdoba, Cordoba; ${ }^{3} \mathrm{MFyC}$ de Valladolid, Area Este, Valladolid, Spain

Background: Dose optimization, such as dose reduction or dose spacing, is nowadays presented as a therapeutic strategy to be followed in patients with rheumatoid arthritis (RA) who have managed to reach and maintain clinical remission for a while. This strategy reduces the frequency of adverse effects and promotes cost savings

Methods: Patients with RA (Criteria ACR 1987) of the CREATE registry (patients who was treated in real life conditions) who had clinical remission (DAS28 <2.6) of at least 6 months of duration on November 1, 2013, constituted the cohort of patients who were optimized for the dose received. According to the consensus of the Spanish Societies of Rheumatology and Hospital Pharmacy, the optimization of doses meant the reduction of between 20 and $50 \%$ of the same.

A multidisciplinary team of rheumatologists and clinical pharmacists in a third-level hospital was involved in decision-making on treatment and dose reduction, which involved the application of protocols and the follow-up of patients at least every two months.

Results: A prospective follow-up of 70 patients with RA who had received optimized doses of biological therapy for 3 years, with a mean age of 56.9 (13.7) years, of which $78.6 \%$ were women, $68.8 \%$ were positive rheumatoid factor and $66.7 \%$ ACPA +

The relapse occurred in $41.8 \%$ (at first year), $56.7 \%$ (at second year) and $62.7 \%$ (at third year). There were no statistical differences between these last 2 percentages. The median survival time of the optimization regimen was 15.24 (4.65) months (95\% Cl: 4.66-25.83). No statistically significant differences were found when comparing patients according to the optimized drug (antiTNF versus non-anti-TNF) (test log.rank: 0.239, p: 0.625).

When relapse occurs, the patient returns to normal doses prior to optimization of the drug. Our data show that $62.7 \%$ of the patients in whom the relapse occurs at 3 years, maintains DAS28 $<2,6(P<0.05)$ when dose was returned to the manufacturer recommended dose.

The $37.3 \%$ (95\% Cl: $26.72 \%, 49.28 \%$ ) patients maintained the optimization pattern throughout the follow-up without relapse, with an average DAS28 of 1.99 $(1.07)$ at 3 years. Comparing these patients with those who relapse, they achieved significantly lower DAS28 values at both ( $p: 0.028)$ and at three years ( $p: 0.025)$

Conclusions:

- The strategy of dose reduction of biological therapies in patients with established RA that achieve sustained remission is possible in $37.3 \%$ of cases in real clinical practice (CREATE Registry) and it was maintained for 3 years.

- The probability of occurrence of relapse decreases after 2 years of treatment with an optimized regimen in those patients who have not relapsed before.

- This strategy is possible in patients with persistently controlled disease and in view of our results, it is independent of the drug administered (antiTNF versus non-antiTNF).

- After 3 years of follow-up, all patients maintained clinical remission (DAS 28 $<2.6$ ) despite relapses, and after resumption of the usual dose, all of them reached the therapeutic goal again.

- Patients who maintain clinical remission for 3 years achieve DAS28 values statistically lower than those who did not after 2 years.

Disclosure of Interest: None declared

DOI: 10.1136/annrheumdis-2017-eular.2156

\section{AB0389 THE IMMUNOGENICITY OF BRANDED AND BIOSIMILAR INFLIXIMAB IN RHEUMATOID ARTHRITIS ACCORDING TO TH9-RELATED RESPONSES}

R. Talotta ${ }^{1}$, A. Berzi ${ }^{2}$, A. Doria ${ }^{3}$, A. Batticciotto ${ }^{1}$, M.C. Ditto ${ }^{1}$, D. Trabattoni ${ }^{1}$, P. Sarzi Puttini ${ }^{1} .{ }^{1}$ Rheumatology Unit; ${ }^{2}$ Department of Biomedical and Clinical Sciences, University Hospital "Luigi Sacco", Milan; ${ }^{3}$ Rheumatology Unit,

University Hospital, Padua, Italy

Background: There are many evidences that Th9 lymphocytes take part to the pathogenesis of rheumatoid arthritis (RA), however it is unclear whether these cells are implicated in the immunogenicity of biologic agents.

Objectives: We aimed to evaluate the immunogenicity of branded and biosimilar infliximab by detecting changes in Th9 percentages following an "in vitro" stimulation test.

Methods: PBMCs from 55 consecutive RA outpatients (15 drug-free, 20 successfully treated with branded infliximab and 20 failing branded infliximab) and from 10 healthy controls were collected. PBMCs were cultured with/without $50 \mu \mathrm{g} / \mathrm{mL}$ infliximab originator (Remicade ${ }^{\circledR}$ ) or $50 \mu \mathrm{g} / \mathrm{mL}$ infliximab biosimilar (Remsima ${ }^{\circledR}$ ), $50 \mu \mathrm{g} / \mathrm{mL}$ Human IgG1kappa and $50 \mu \mathrm{g} / \mathrm{mL}$ recombinant Human IgG Fc for 18 hours. Th9 lymphocytes were identified by means of flow cytometry as PU.1+, IRF4+, IL9+ CD4+ cells. Furthermore, the markers CCR7 and CD45RA were used to distinguish naive from memory IL-9-producer cells.

Results: In unstimulated condition, RA patients showed the highest percentages of Th9 lymphocytes. Following stimulation with branded infliximab, the percentage of PU.1+, IRF4+ Th9 cells, CCR7+, CD45RA- central memory and CCR7-, CD45RA- effector memory IL-9 producer cells significantly increased in the group of infliximab non responder RA patients. On the contrary, no significant variation was observed after biosimilar exposure. Figures 1,2,3,4 resume the results.

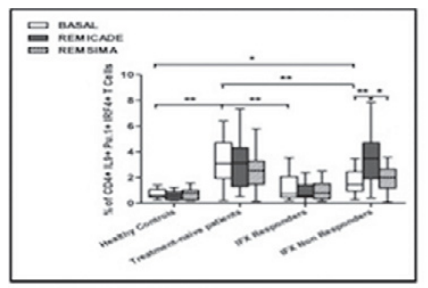

Fig.1 Overall Th9 cells

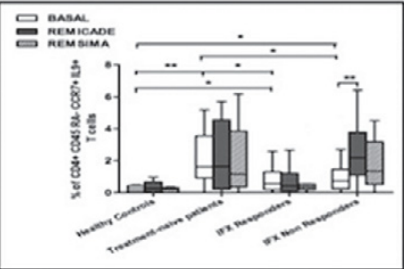

Fig. 3 Central memory Th9 cells

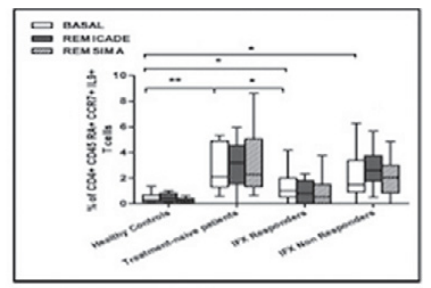

Fig. 2 Naive Th9 cells

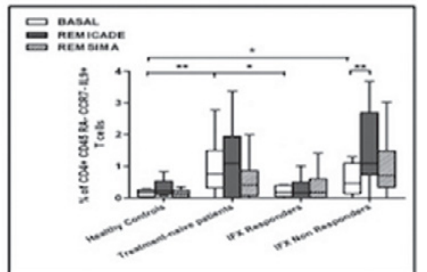

Fig.4 Effector memory Th9 cells
Conclusions: According to our results, Th9 cells seem to be involved in the immune response against the epitopes of branded but not biosimilar infliximab and this condition could rely on the recall and the stimulation of both central and effector memory cells. Further studies are indeed needed to confirm these data. Disclosure of Interest: None declared DOI: 10.1136/annrheumdis-2017-eular.4401 